\title{
Option pricing under a stressed-beta model
}

\author{
Jean-Pierre Fouque · Adam P. Tashman
}

Received: 2 June 2009 / Accepted: 22 October 2009 / Published online: 10 November 2009

(C) The Author(s) 2009. This article is published with open access at Springerlink.com

\begin{abstract}
Empirical studies have concluded that stochastic volatility is an important component of option prices. We introduce a regime-switching mechanism into a continuous-time Capital Asset Pricing Model which naturally induces stochastic volatility in the asset price. Under this Stressed-Beta model, the mechanism is relatively simple: the slope coefficient-which measures asset returns relative to market returns - switches between two values, depending on the market being above or below a given level. After specifying the model, we use it to price European options on the asset. Interestingly, these option prices are given explicitly as integrals with respect to known densities. We find that the model is able to produce a volatility skew, which is a prominent feature in option markets. This opens the possibility of forward-looking calibration of the slope coefficients, using option data, as illustrated in the paper.
\end{abstract}

Keywords Stressed-beta model C CAPM - Stochastic volatility · Regime-switching · Option pricing $\cdot$ Implied volatility skews $\cdot$ Calibration

JEL Classification $\quad \mathrm{C} 02 \cdot \mathrm{G} 13$

\section{Introduction}

The concept of stock betas was developed in the context of the Capital Asset Pricing Model of Treynor (1962), Sharpe (1964), Lintner (1965), and Mossin (1966) and was

Work of J.-P. Fouque was partially supported by NSF grant DMS-0806461.

J.-P. Fouque ( $\varangle)$ · A. P. Tashman

Department of Statistics and Applied Probability, University of California, Santa Barbara,

CA 93106-3110, USA

e-mail: fouque@pstat.ucsb.edu

A. P. Tashman

e-mail: tashman@pstat.ucsb.edu 
based on previous portfolio theory in Markowitz (1952). The beta of a stock represents the scale of the risk of the asset relative to the systematic risk of the market and is critical in the development and performance of stock portfolios. It is now accepted that this linear model does not perform well empirically, and much research has been devoted to its improvement.

One popular approach to extending CAPM has been to retain linearity in the model, but to consider a beta which changes over time (Ferson 1989; Ferson and Harvey 1991, 1993; Ferson and Korajczyk 1995; Jagannathan and Wang 1996). These papers argue that allowing a dynamic beta should improve the model. However, Ghysels (1998) finds that betas change very slowly over time, and linear factor models may overstate the time variation.

In Fouque and Kollman (2009), we proposed a "forward-looking" approach to the calibration of a constant beta-parameter in a continuous-time CAPM model which includes a fast mean-reverting stochastic volatility component in the dynamics of the market price.

In the present paper, we no longer assume beta constant but rather follow a second approach which consists in introducing nonlinearity through a state-switching mechanism. In Fridman (1994), a two-state CAPM model is proposed in which the excess returns for the market and a particular security are bivariate normally distributed. The parameters of the distribution are determined by an unobservable Markov chain, where the two states represent business regimes of low and high volatility. The threshold CAPM model introduced in Akdeniz et al. (2003) expresses market risk as a function of an underlying economic variable termed a threshold variable. When the threshold variable is at or below a level $\lambda$, the beta takes value $\beta_{1}$, and when it is above $\lambda$, it takes value $\beta_{2}$. Formulated in this fashion, threshold CAPM treats beta as constant so long as the threshold variable remains on one side of the boundary. This discrete-time model outperforms the CAPM model, lending support for its realistic approximation of beta.

This paper examines the Stressed-Beta model, which can be regarded as a continuous-time threshold CAPM model using the stock market as the threshold variable. Stressed-Beta models have been introduced in the context of hedge fund risk management in discrete time in Tashman and Frey (2009), and in continuous time in Tashman (2009). In fact, in a Stressed-Beta model, the volatility of the stock becomes stochastic, driven by the market. The model can be seen as a volatility regime-switching model, or a stochastic volatility model. However, the market is complete when trading in the stock and in the market, so that the risk-neutral pricing measure is unique as shown in Sect. 3. Also, the correlation structure (leverage effect) is very particular in this model, and induces a negative correlation ("market goes up, stock volatility goes down").

Stochastic volatility models form a rich class of models which, in particular, generate the observed skews of implied volatilities [see for instance Fouque et al. (2000)]. In general, unlike in the Black-Scholes model, there is no explicit option pricing formula, one exception being the Heston model (Heston 1993) for which European options are given semi-explicitly, up to an inverse Fourier transform.

Surprisingly, we show that our regime-switching stressed-beta model leads to an explicit formula for pricing a European option (up to computation of integrals of known 
densities). The derivation of this formula, presented in Sect. 4, relies on passage time analysis for Brownian motions and on the joint distribution of the triplet (terminal value, local time, occupation time) derived in Karatzas and Shreve (1984, 1991).

In Sect. 5, we show how to incorporate in the model a fast mean-reverting stochastic volatility component in the dynamics of the market price, in order to account for the skew of implied volatility in the options on the market as well. We use a singular perturbation method introduced in Fouque et al. (2000) and also employed in Fouque and Kollman (2009).

In Sect. 6, we present implied volatility skews generated by call option prices computed with the formula (35) derived in Sect. 4.3, and a calibration example.

\section{The stressed-beta continuous-time CAPM model}

\subsection{The continuous-time CAPM model}

To start, we consider a simple continuous-time CAPM model in which the market price $M_{t}$ and an asset price $S_{t}$ evolve as follows:

$$
\begin{aligned}
\frac{d M_{t}}{M_{t}} & =\mu d t+\sigma_{m} d W_{t}, \\
\frac{d S_{t}}{S_{t}} & =\beta \frac{d M_{t}}{M_{t}}+\sigma d Z_{t},
\end{aligned}
$$

for constant positive volatilities $\sigma_{m}$ and $\sigma$, and a slope $\beta$. This model is consistent with CAPM in that that the return of the asset $\frac{d S_{t}}{S_{t}}$ is a linear function of the return of the market $\frac{d M_{t}}{M_{t}}$ through the $\beta$ coefficient and a Brownian-driven noise process. In this model, under the physical probability measure $\mathbb{P}$, we assume independence between the standard Brownian motions driving the market and asset price processes:

$$
d\langle W, Z\rangle_{t}=0 \text {. }
$$

Most importantly, the process preserves the definition of the $\beta$ coefficient as the covariance of the asset and market returns divided by the market variance, that is formally:

$$
\begin{aligned}
\frac{\operatorname{Cov}\left(\frac{d S_{t}}{S_{t}}, \frac{d M_{t}}{M_{t}}\right)}{\operatorname{Var}\left(\frac{d M_{t}}{M_{t}}\right)}= & \frac{\operatorname{Cov}\left(\beta \frac{d M_{t}}{M_{t}}+\sigma d Z_{t}, \frac{d M_{t}}{M_{t}}\right)}{\operatorname{Var}\left(\frac{d M_{t}}{M_{t}}\right)} \\
& =\frac{\operatorname{Cov}\left(\beta \frac{d M_{t}}{M_{t}}, \frac{d M_{t}}{M_{t}}\right)}{\operatorname{Var}\left(\frac{d M_{t}}{M_{t}}\right)}=\beta,
\end{aligned}
$$

where the second equality holds due to the independence of $M_{t}$ and $Z_{t}$. Observe that the evolution of $S_{t}$ is given by

$$
\frac{d S_{t}}{S_{t}}=\beta \mu d t+\beta \sigma_{m} d W_{t}+\sigma d Z_{t},
$$

that is a geometric Brownian motion with volatility $\sqrt{\beta^{2} \sigma_{m}^{2}+\sigma^{2}}$. 
Therefore, in terms of options on the asset, this model is nothing else than the constant volatility Black-Scholes model which cannot capture the observed skew of implied volatilities in option data.

Fouque and Kollman (2009) introduced a continuous-time CAPM model with stochastic volatility in which $\sigma_{m}$ is driven by an additional stochastic processes. Here, we generalize the continuous-time CAPM model in a different direction, namely in the case where the slope $\beta$ is no longer a constant, but depends on $M_{t}$.

\subsection{The stressed-beta model}

We extend the CAPM model by considering a piecewise-linear relationship between the asset and the market. When the market is above a given level $c>0$ (this may be the case when the economy is in a good regime), the slope takes the value $\beta$, but when the market is below this level $c$ (the bad regime), the slope switches to the value $\beta+\delta$. Generally, $\delta$ will be positive, thus the slope will be steeper when $M_{t}<c$. The slope is written as

$$
\beta\left(M_{t}\right)=\beta+\delta \mathbf{1}_{\left\{M_{t}<c\right\}},
$$

where $\mathbf{1}_{A}$ denotes the indicator function of the set $A$.

Given (5), the model for the market price $M_{t}$ and the asset price $S_{t}$ evolve as follows:

$$
\begin{aligned}
\frac{d M_{t}}{M_{t}} & =\mu d t+\sigma_{m} d W_{t}, \\
\frac{d S_{t}}{S_{t}} & =\beta\left(M_{t}\right) \frac{d M_{t}}{M_{t}}+\sigma d Z_{t},
\end{aligned}
$$

where, as before, the volatilities $\sigma_{m}$ and $\sigma$ are constant, and $W_{t}$ and $Z_{t}$ are independent Brownian motions (3). This model preserves the definition of $\beta$ as the covariance of the asset and market returns divided by the variance of the market, since, given $M_{t}$, the computation (4) remains the same with $\beta$ replaced by $\beta\left(M_{t}\right)$.

Substituting the market equation (6) into the asset equation (7) yields

$$
\frac{d S_{t}}{S_{t}}=\beta\left(M_{t}\right) \mu d t+\beta\left(M_{t}\right) \sigma_{m} d W_{t}+\sigma d Z_{t},
$$

which appears as a stochastic volatility model through the slope-switching mechanism $\beta\left(M_{t}\right)$ driven by the market price level $M_{t}$.

\section{Option pricing in the stressed-beta model}

Consider a European option written on the stock $S$ with maturity date $T$, and payoff function $h(S)$. In this paper we assume that the risk free rate $r$ is constant. The problem of option pricing can be approached in two different ways: risk-neutral valuation or 
replication. In both approaches, it is essential to keep in mind that the market $M$ and the asset $S$ are both tradable.

\subsection{Risk-neutral pricing}

The market (or index) and the asset being both tradable, their discounted prices need to be martingales under a risk-neutral pricing measure. Recall that $\left(W_{t}, Z_{t}\right)$ are two independent standard Brownian motions, and rewrite the system $(6,7)$ as:

$$
\begin{aligned}
\frac{d M_{t}}{M_{t}} & =r d t+\sigma_{m}\left(d W_{t}+\frac{\mu-r}{\sigma_{m}} d t\right) \\
\frac{d S_{t}}{S_{t}} & =r d t+\beta\left(M_{t}\right) \sigma_{m}\left(d W_{t}+\frac{\mu-r}{\sigma_{m}} d t\right)+\sigma\left(d Z_{t}+\frac{\left(\beta\left(M_{t}\right)-1\right) r}{\sigma} d t\right) .
\end{aligned}
$$

We set

$$
\begin{aligned}
d W_{t}^{*} & =d W_{t}+\frac{\mu-r}{\sigma_{m}} d t, \\
d Z_{t}^{*} & =d Z_{t}+\frac{\left(\beta\left(M_{t}\right)-1\right) r}{\sigma} d t,
\end{aligned}
$$

and we observe that the ratios $\frac{\mu-r}{\sigma_{m}}$ and $\frac{\left(\beta\left(M_{t}\right)-1\right) r}{\sigma}$ are bounded. By Girsanov theorem, there is a unique equivalent probability $\mathbb{P}^{\star} \sim \mathbb{P}$ such that $\left(W_{t}^{*}, Z_{t}^{*}\right)$ are independent standard Brownian motions under $\mathbb{P}^{\star}$, called the pricing equivalent martingale measure or risk-neutral measure. Under $\mathbb{P}^{\star}$, the dynamics $(6,7)$ becomes:

$$
\begin{aligned}
& \frac{d M_{t}}{M_{t}}=r d t+\sigma_{m} d W_{t}^{*}, \\
& \frac{d S_{t}}{S_{t}}=r d t+\beta\left(M_{t}\right) \sigma_{m} d W_{t}^{*}+\sigma d Z_{t}^{*} .
\end{aligned}
$$

By the classical no-arbitrage argument, the price of the option at time $t<T$, denoted by $P_{t}$, is then given by

$$
P_{t}=\mathbb{E}^{\star}\left\{e^{-r(T-t)} h\left(S_{T}\right) \mid \mathcal{F}_{t}\right\}=P\left(t, M_{t}, S_{t}\right),
$$

where $\mathcal{F}_{t}$ denotes the filtration generated by $\left(M_{t}, S_{t}\right)$, or equivalently by the two Brownian motions, and we have used the Markov property of $\left(M_{t}, S_{t}\right)$ to write the price of the option as a function of $\left(t, M_{t}, S_{t}\right)$.

\subsection{Replication and pricing PDE}

The derivation of an adapted, replicating, and self-financing strategy follows the lines of the original Black-Scholes-Merton derivation with, in the present case, two tradable risky assets $M$ and $S$. One seeks a portfolio made, at time $t$, of $a_{t}$ shares of market 
$M, b_{t}$ shares of asset $S$, and $c_{t} e^{r t}$ in cash, such that it replicates the price of the option

$$
a_{t} M_{t}+b_{t} S_{t}+c_{t} e^{r t}=P\left(t, M_{t}, S_{t}\right), \quad t \leq T,
$$

and it is self-financing

$$
a_{t} d M_{t}+b_{t} d S_{t}+r c_{t} e^{r t} d t=d P\left(t, M_{t}, S_{t}\right)
$$

Using Itô's formula and canceling the risks from the Brownian motions $W_{t}$ and $Z_{t}$, one finds that

$$
a_{t}=\frac{\partial P}{\partial M}\left(t, M_{t}, S_{t}\right), \quad b_{t}=\frac{\partial P}{\partial S}\left(t, M_{t}, S_{t}\right)
$$

where $P(t, M, S)$ satisfies the pricing partial differential equation with terminal condition:

$$
\begin{gathered}
\frac{\partial P}{\partial t}+\frac{1}{2} \sigma_{m}^{2} M^{2} \frac{\partial^{2} P}{\partial M^{2}}+\frac{1}{2}\left(\sigma_{m}^{2} \beta^{2}(M)+\sigma^{2}\right) S^{2} \frac{\partial^{2} P}{\partial S^{2}}+\beta(M) \sigma_{m}^{2} M S \frac{\partial^{2} P}{\partial M \partial S} \\
+r\left(M \frac{\partial P}{\partial M}+S \frac{\partial P}{\partial S}-P\right)=0, \\
P(T, M, S)=h(S) .
\end{gathered}
$$

Indeed, (11) is the Feynman-Kac representation of the solution to the problem (13-14).

\section{Option pricing formula}

In this section, we show that, surprisingly, the option price given by (11) admits a closed-form solution as an integral with respect to a multidimensional known density. Since the derivation of this formula is purely probabilistic, for simplicity we consider the case where $t=0$, so that the time-to-maturity is simply $T-t=T$.

\subsection{Log-variables and driftless market}

We first consider the $\log$-variables $\xi_{t}=\log M_{t}$ and $X_{t}=\log S_{t}$, so that the riskneutral dynamics (9-10) become:

$$
\begin{aligned}
d \xi_{t} & =\left(r-\frac{\sigma_{m}^{2}}{2}\right) d t+\sigma_{m} d W_{t}^{*} \\
d X_{t} & =\left(r-\frac{1}{2}\left(\sigma_{m}^{2} \beta^{2}\left(e^{\xi_{t}}\right)+\sigma^{2}\right)\right) d t+\beta\left(e^{\xi_{t}}\right) \sigma_{m} d W_{t}^{*}+\sigma d Z_{t}^{*}
\end{aligned}
$$


In integral form, starting from the initial point $\xi_{0}=\xi$, (15) becomes:

$$
\xi_{t}=\xi+\left(r-\frac{\sigma_{m}^{2}}{2}\right) t+\sigma_{m} W_{t}^{*}
$$

In integral form, starting from the initial point $X_{0}=x$ and evaluated at time $T,(16)$ becomes:

$$
\begin{aligned}
X_{T}= & x+\left(r-\frac{\sigma^{2}}{2}\right) T-\frac{\sigma_{m}^{2}}{2} \int_{0}^{T} \beta^{2}\left(e^{\xi_{t}}\right) d t+\sigma_{m} \int_{0}^{T} \beta\left(e^{\xi_{t}}\right) d W_{t}^{*}+\sigma Z_{T}^{*} \\
= & x+\left(r-\frac{\sigma_{m}^{2} \beta^{2}+\sigma^{2}}{2}\right) T+\sigma_{m} \beta W_{T}^{*}+\sigma Z_{T}^{*} \\
& -\left(\delta^{2}+2 \delta \beta\right) \frac{\sigma_{m}^{2}}{2} \int_{0}^{T} \mathbf{1}_{\left\{\xi_{t}<\log c\right\}} d t+\sigma_{m} \delta \int_{0}^{T} \mathbf{1}_{\left\{\xi_{t}<\log c\right\}} d W_{t}^{*},
\end{aligned}
$$

where we have used the particular form (5) for the function $\beta(M)$ :

$$
\beta(M)=\beta+\delta \mathbf{1}_{\{M<c\}}
$$

The expression (18) involves the integral $\int_{0}^{T} \mathbf{1}_{\left\{\xi_{t}<\log c\right\}} d t$, which is the occupation time of $\xi_{t}$, the Brownian motion with drift given by (17). Our next step is to remove the drift by a Girsanov change of probability. Consider the new probability measure $\widetilde{\mathbb{P}}$ defined on $\mathcal{F}_{T}$ by

$$
\begin{aligned}
\frac{d \widetilde{\mathbb{P}}}{d \mathbb{P}^{\star}} & =\exp \left\{-\theta W_{T}^{*}-\frac{1}{2} \theta^{2} T\right\}, \\
\theta & =\frac{1}{\sigma_{m}}\left(r-\frac{\sigma_{m}^{2}}{2}\right) .
\end{aligned}
$$

Setting

$$
\widetilde{W}_{t}=W_{t}^{*}+\theta t, \quad \widetilde{Z}_{t}=Z_{t}^{*},
$$

then under $\widetilde{\mathbb{P}}$, the processes $\widetilde{W}_{t}$ and $\widetilde{Z}_{t}$ are two independent standard Brownian motions, and (17) becomes the driftless Brownian motion:

$$
\xi_{t}=\xi+\sigma_{m} \widetilde{W}_{t}
$$


Consequently, (18) becomes

$$
\begin{aligned}
X_{T}= & x+A_{1} T+\sigma_{m} \beta \widetilde{W}_{T}+\sigma \widetilde{Z}_{T}^{T} \\
& -A_{2} \int_{0}^{T} \mathbf{1}_{\left\{\xi_{t}<\log c\right\}} d t+\sigma_{m} \delta \int_{0}^{T} \mathbf{1}_{\left\{\xi_{t}<\log c\right\}} d \widetilde{W}_{t},
\end{aligned}
$$

where $A_{1}$ and $A_{2}$ are constants defined as

$$
\begin{aligned}
& A_{1}=r(1-\beta)-\frac{\sigma_{m}^{2}\left(\beta^{2}-\beta\right)+\sigma^{2}}{2}, \\
& A_{2}=\delta(\delta+2 \beta-1) \frac{\sigma_{m}^{2}}{2}+\delta r .
\end{aligned}
$$

4.2 Hitting time and conditional distribution of $X_{T}$

Next, we introduce the first passage time

$$
\tau=\inf \left\{t \geq 0: \xi_{t}=\log c\right\}=\inf \left\{t \geq 0: \widetilde{W}_{t}=\tilde{c}\right\}
$$

where we have used (19) for $\xi_{t}$ under $\widetilde{\mathbb{P}}$ and the notation

$$
\tilde{c}=\frac{\log c-\xi}{\sigma_{m}} .
$$

Using the stopping time $\tau \wedge T$, (20) can be rewritten

$$
\begin{aligned}
X_{T}= & x+A_{1} T+\sigma_{m} \beta \widetilde{W}_{T}+\sigma \widetilde{Z}_{T} \\
& -A_{2}(\tau \wedge T) \mathbf{1}_{\{\tilde{c}>0\}}-A_{2} \int_{\tau \wedge T}^{T} \mathbf{1}_{\left\{\widetilde{W}_{t}<\tilde{c}\right\}} d t \\
& +\sigma_{m} \delta \widetilde{W}_{\tau \wedge T} \mathbf{1}_{\{\tilde{c}>0\}}+\sigma_{m} \delta \int_{\tau \wedge T}^{T} \mathbf{1}_{\left\{\widetilde{W}_{t}<\tilde{c}\right\}} d \widetilde{W}_{t} .
\end{aligned}
$$

The stochastic integral appearing in (23) can be re-expressed in terms of the local time $\widetilde{L}^{\tilde{c}}$ of $\widetilde{W}$ at level $\tilde{c}$. Applying Tanaka's formula (see Karatzas and Shreve 1991, Section 3.6) to the function $\phi(w)=(w-\tilde{c}) \mathbf{1}_{\{w<\tilde{c}\}}$ between $\tau \wedge T$ and $T$, we get:

$$
\int_{\tau \wedge T}^{T} \mathbf{1}_{\left\{\widetilde{W}_{t}<\tilde{c}\right\}} d \widetilde{W}_{t}=\phi\left(\widetilde{W}_{T}\right)-\phi\left(\widetilde{W}_{\tau \wedge T}\right)+\widetilde{L}_{T}^{\tilde{c}}-\widetilde{L}_{\tau \wedge T}^{\tilde{c}} .
$$


If $\xi=\log c$, or equivalently $\tilde{c}=0$, then $\tau=0$.

If $\xi \neq \log c$, or equivalently $\tilde{c} \neq 0$, then the probability distribution of $\tau \wedge T$ is given by

$$
p(u ; \tilde{c}) \mathbf{1}_{(0, T)}(u) d u+\widetilde{\mathbb{P}}\{\tau>T\} \delta_{T}(d u),
$$

where the density $p(u ; \tilde{c})$ is given by (Karatzas and Shreve 1991, Sect. 2.6.C):

$$
p(u ; \tilde{c})=\frac{|\tilde{c}|}{\sqrt{2 \pi u^{3}}} \exp \left(-\frac{\tilde{c}^{2}}{2 u}\right), \quad u>0,
$$

and

$$
\widetilde{\mathbb{P}}\{\tau>T\}=\int_{T}^{\infty} p(u ; \tilde{c}) d u=2 N_{T}(|\tilde{c}|)-1,
$$

where $N_{T}$ denotes the $\mathcal{N}(0, T)$-cdf.

At this point, it is convenient to treat separately the cases $\xi=\log c, \xi>\log c$, and $\xi<\log c$ (or equivalently $\tilde{c}=0, \tilde{c}<0$ and $\tilde{c}>0$ respectively).

Case $\xi=\log c$

In that case, $\tau=0$, and from (23) and (24) we get:

$$
\begin{aligned}
X_{T}= & x+A_{1} T+\sigma_{m} \beta \widetilde{W}_{T}+\sigma \widetilde{Z}_{T} \\
& -A_{2} \int_{0}^{T} \mathbf{1}_{\left\{\widetilde{W}_{t}<0\right\}} d t+\sigma_{m} \delta\left(\widetilde{W}_{T} \mathbf{1}_{\left\{\widetilde{W}_{T}<0\right\}}+\widetilde{L}_{T}^{0}\right) \\
= & x+\left(A_{1}-A_{2}\right) T+\sigma \widetilde{Z}_{T} \\
& +A_{2} \int_{0}^{T} \mathbf{1}_{\left\{\widetilde{W}_{t}>0\right\}} d t+\sigma_{m} \widetilde{W}_{T}\left(\beta+\delta \mathbf{1}_{\left\{\widetilde{W}_{T}<0\right\}}\right)+\sigma_{m} \delta \widetilde{L}_{T}^{0} \\
= & : \Psi_{0}\left(\widetilde{W}_{T}, \widetilde{L}_{T}^{0}, \widetilde{\Gamma}_{T}^{+}, \widetilde{Z}_{T}\right),
\end{aligned}
$$

where we have also expressed the occupation time of $(-\infty, 0)$ in terms of the occupation time of $(0, \infty)$ denoted by $\widetilde{\Gamma}_{T}^{+}=\int_{0}^{T} \mathbf{1}_{\left\{\tilde{W}_{t}>0\right\}} d t$. It is now clear that the distribution of $X_{T}$ is given explicitly in terms of the distribution of the triplet $\left(\widetilde{W}_{T}, \widetilde{L}_{T}^{0}, \widetilde{\Gamma}_{T}^{+}\right)$and the independent Gaussian random variable $\widetilde{Z}_{T}$. The density of the triplet is derived in Karatzas and Shreve (1984) (see also Karatzas and Shreve 1991, Section 6.3.C): 


$$
\begin{aligned}
\mathbb{P} & \left\{\widetilde{W}_{T} \in d a, \widetilde{L}_{T}^{0} \in d b, \widetilde{\Gamma}_{T}^{+} \in d \gamma\right\} \\
& =\left\{\begin{array}{l}
2 p(T-\gamma ; b) p(\gamma ; a+b) \\
2 p(\gamma ; b) p(T-\gamma ;-a+b) \text { if } a<0, b>0,0<\gamma<T,
\end{array}\right.
\end{aligned}
$$

where $p(u ; \cdot)$ is given by $(25)$.

Case $\xi<\log c$

In that case, $\tilde{c}>0$, and from (23) and (24) we get:

$$
\begin{aligned}
X_{T}= & x+A_{1} T+\sigma_{m} \beta \widetilde{W}_{T}+\sigma \widetilde{Z}_{T} \\
& -A_{2}(\tau \wedge T)-A_{2} \int_{\tau \wedge T}^{T} \mathbf{1}_{\left\{\widetilde{W}_{t}<\tilde{c}\right\}} d t+\sigma_{m} \delta \widetilde{W}_{\tau \wedge T} \\
& +\sigma_{m} \delta\left[\left(\widetilde{W}_{T}-\tilde{c}\right) \mathbf{1}_{\left\{\tilde{W}_{T}<\tilde{c}\right\}}-\left(\widetilde{W}_{\tau \wedge T}-\tilde{c}\right) \mathbf{1}_{\left\{\tilde{W}_{\tau \wedge T}<\tilde{c}\right\}}+\widetilde{L}_{T}^{\tilde{c}}-\widetilde{L}_{\tau \wedge T}^{\tilde{c}}\right] .
\end{aligned}
$$

- On $\{\tau>T\}$, we have:

$$
\begin{aligned}
X_{T} & =x+\left(A_{1}-A_{2}\right) T+\sigma_{m}(\beta+\delta) \widetilde{W}_{T}+\sigma \widetilde{Z}_{T} \\
& =: \Psi_{T^{+}}^{-}\left(\widetilde{W}_{T}, \widetilde{Z}_{T}\right),
\end{aligned}
$$

where the upper index "-" stands for $\xi$ below the level $\log c$, and the lower index $T^{+}$ stands for $\tau>T$.

Therefore, in this case, the distribution of $X_{T}$ is given by the distribution of the independent Gaussian random variable $\widetilde{Z}_{T}$, and the conditional distribution of $\widetilde{W}_{T}$ given that $\{\tau>T\}$. From Karatzas and Shreve (1991), Section 2.8.A, one easily obtains:

$$
\begin{aligned}
\mathbb{P}\left\{\widetilde{W}_{T} \in d a, \tau>T\right\} & =\frac{1}{\sqrt{2 \pi T}}\left(e^{-\frac{a^{2}}{2 T}}-e^{-\frac{(2 \tilde{c}-a)^{2}}{2 T}}\right) d a, \quad a<\tilde{c} \\
& =: q_{T}(a ; \tilde{c}) d a .
\end{aligned}
$$

- On $\{\tau=u\}$ with $u \leq T$, we have $\widetilde{W}_{u}=\tilde{c}$, and $X_{T}$ is given by

$$
\begin{aligned}
X_{T}= & x+\left(A_{1}-A_{2}\right) T+\sigma_{m}(\beta+\delta) \tilde{c}+\sigma_{m} \beta\left(\widetilde{W}_{T}-\widetilde{W}_{u}\right)+\sigma \widetilde{Z}_{T} \\
& +A_{2} \int_{u}^{T} \mathbf{1}_{\left\{\widetilde{W}_{t}-\widetilde{W}_{u}>0\right\}} d t \\
& +\sigma_{m} \delta\left[\left(\widetilde{W}_{T}-\widetilde{W}_{u}\right) \mathbf{1}_{\left\{\widetilde{W}_{T}-\widetilde{W}_{u}<0\right\}}+\widetilde{L}_{T}^{\tilde{c}}-\widetilde{L}_{u}^{\tilde{c}}\right] .
\end{aligned}
$$

Therefore, in this case, the distribution of $X_{T}$ is given by the distribution of $\widetilde{Z}_{T}$ and an independent triplet $\left(B_{T-u}, L_{T-u}^{0}, \Gamma_{T-u}^{+}\right)$formed by the value, the local time 
at 0 , and the occupation time of the positive half-space, at time $T-u$, of a standard Brownian motion $B$. That is, in distribution:

$$
\begin{aligned}
X_{T}= & x+\left(A_{1}-A_{2}\right) T+\sigma_{m}(\beta+\delta) \tilde{c}+\sigma_{m} B_{T-u}\left(\beta+\delta \mathbf{1}_{\left\{B_{T-u}<0\right\}}\right)+\sigma \widetilde{Z}_{T} \\
& +A_{2} \Gamma_{T-u}^{+}+\sigma_{m} \delta L_{T-u}^{0} \\
=: & \Psi_{T^{-}}^{-}\left(B_{T-u}, L_{T-u}^{0}, \Gamma_{T-u}^{+}, \widetilde{Z}_{T}\right) .
\end{aligned}
$$

The distribution of the triplet $\left(B_{T-u}, L_{T-u}^{0}, \Gamma_{T-u}^{+}\right)$is given by (28) with $T$ replaced by $T-u$.

Case $\xi>\log c$

In that case, $\tilde{c}<0$, and from (23) and (24) we get:

$$
\begin{aligned}
X_{T}= & x+A_{1} T+\sigma_{m} \beta \widetilde{W}_{T}+\sigma \widetilde{Z}_{T}-A_{2} \int_{\tau \wedge T}^{T} \mathbf{1}_{\left\{\widetilde{W}_{t}<\tilde{c}\right\}} d t \\
& +\sigma_{m} \delta\left[\left(\widetilde{W}_{T}-\tilde{c}\right) \mathbf{1}_{\left\{\tilde{W}_{T}<\tilde{c}\right\}}-\left(\widetilde{W}_{\tau \wedge T}-\tilde{c}\right) \mathbf{1}_{\left\{\tilde{W}_{\tau \wedge T}<\tilde{c}\right\}}+\widetilde{L}_{T}^{\tilde{c}}-\widetilde{L}_{\tau \wedge T}^{\tilde{c}}\right] .
\end{aligned}
$$

- On $\{\tau>T\}$, we have:

$$
\begin{aligned}
X_{T} & =x+A_{1} T+\beta \sigma_{m} \widetilde{W}_{T}+\sigma \widetilde{Z}_{T} \\
& =: \Psi_{T^{+}}^{+}\left(\widetilde{W}_{T}, \widetilde{Z}_{T}\right),
\end{aligned}
$$

where $\widetilde{Z}_{T}$ and $\widetilde{W}_{T}$ are independent and the distribution of $\widetilde{W}_{T}$ is given by (30) with $a>\tilde{c}$ in this case.

- On $\{\tau=u\}$ with $u \leq T$, we have $\widetilde{W}_{u}=\tilde{c}$, and $X_{T}$ is given by

$$
\begin{aligned}
X_{T}= & x+A_{1} T+\sigma_{m} \beta \tilde{c}+\sigma_{m} \beta\left(\widetilde{W}_{T}-\widetilde{W}_{u}\right)+\sigma \widetilde{Z}_{T} \\
& -A_{2} \int_{u}^{T} \mathbf{1}_{\left\{\widetilde{W}_{t}-\widetilde{W}_{u}<0\right\}} d t+\sigma_{m} \delta\left[\left(\widetilde{W}_{T}-\widetilde{W}_{u}\right) \mathbf{1}_{\left\{\tilde{W}_{T}-\widetilde{W}_{u}<0\right\}}+\widetilde{L}_{T}^{\tilde{c}}-\widetilde{L}_{u}^{\tilde{c}}\right] .
\end{aligned}
$$

Therefore, in this case the distribution of $X_{T}$ is given by the distribution of $\widetilde{Z}_{T}$ and an independent triplet $\left(B_{T-u}, L_{T-u}^{0}, \Gamma_{T-u}^{-}\right)$formed by the value, the local time at 0 , and the occupation time of the negative half-space, at time $T-u$, of a standard Brownian motion $B$. That is, in distribution:

$$
\begin{aligned}
X_{T}= & x+A_{1} T+\sigma_{m} \beta \tilde{c}++\sigma_{m} B_{T-u}\left(\beta+\delta \mathbf{1}_{\left\{B_{T-u}<0\right\}}\right)+\sigma \widetilde{Z}_{T} \\
& -A_{2} \Gamma_{T-u}^{-}+\sigma_{m} \delta L_{T-u}^{0} \\
=: & \Psi_{T^{-}}^{+}\left(B_{T-u}, L_{T-u}^{0}, \Gamma_{T-u}^{-}, \widetilde{Z}_{T}\right) .
\end{aligned}
$$


The distribution of the triplet $\left(B_{T-u}, L_{T-u}^{0}, \Gamma_{T-u}^{-}\right)$is the same as the distribution of the triplet $\left(-B_{T-u}, L_{T-u}^{0}, T-u-\Gamma_{T-u}^{-}\right)$, given by (28) with $(a, T, \gamma)$ replaced by $(-a, T-u, T-u-\gamma)$.

\subsection{Pricing formula}

From (11) and the change of measure introduced in Sect. 4.1, the price at time $t=0$, starting from $\left(M_{0}, S_{0}\right)=\left(e^{\xi}, e^{x}\right)$, of a European option with payoff $h\left(S_{T}\right)$ at maturity $T$ is given by

$$
\begin{aligned}
P_{0} & =\mathbb{E}^{\star}\left\{e^{-r T} h\left(S_{T}\right)\right\} \\
& =\widetilde{\mathbb{E}}\left\{e^{-r T} h\left(e^{X_{T}}\right) \frac{d \mathbb{P}^{\star}}{d \widetilde{\mathbb{P}}}\right\} \\
& =\widetilde{\mathbb{E}}\left\{e^{-r T} h\left(e^{X_{T}}\right) e^{\theta \widetilde{W}_{T}-\frac{1}{2} \theta^{2} T}\right\} \\
& =e^{-r T} e^{-\frac{1}{2} \theta^{2} T} \widetilde{\mathbb{E}}\left\{h\left(e^{X_{T}}\right) e^{\theta \widetilde{W}_{T}}\right\} .
\end{aligned}
$$

Decomposing the expectation on $\{\tau \leq T\}$ and $\{\tau>T\}$, one obtains:

$$
\begin{aligned}
P_{0}= & e^{-\left(r+\frac{1}{2} \theta^{2}\right) T}\left[\int_{0}^{T} \widetilde{\mathbb{E}}\left\{h\left(e^{X_{T}}\right) e^{\theta\left(\widetilde{W}_{T}-\widetilde{W}_{u}\right)+\theta \tilde{c}} \mid \tau=u\right\} p(u ; \tilde{c}) d u\right. \\
& \left.+\widetilde{\mathbb{E}}\left\{h\left(e^{X_{T}}\right) e^{\theta \widetilde{W}_{T}} \mathbf{1}_{\{\tau>T\}}\right\}\right],
\end{aligned}
$$

where the density $p(u ; \tilde{c})$ is given by (25) and the degenerate case $\tilde{c}=0$ (or $\xi=\log c$ ) corresponds to $p(u ; 0) d u=\delta_{0}(d u)$.

Using the conditional distributions derived in Sect. 4.2 and denoting by $n_{T}(z)$ the $\mathcal{N}(0, T)$ density, we get:

$$
\begin{aligned}
P_{0}= & e^{-\left(r+\frac{1}{2} \theta^{2}\right) T}\left[e^{\theta \tilde{c}} \int_{-\infty}^{\infty} \int_{0}^{T} \int_{0}^{T-u} \int_{0}^{\infty} \int_{-\infty}^{\infty} h\left(e^{\Psi_{T^{-}}^{ \pm}(a, b, \gamma, z)}\right) e^{\theta a}\right. \\
& \times g(a, b, \gamma ; T-u) d a d b d \gamma p(u ; \tilde{c}) d u n_{T}(z) d z \\
& \left.+\left(\int_{-\infty}^{\infty} \int_{D^{ \pm}} h\left(e^{\Psi_{T^{+}}^{ \pm}(a, z)}\right) e^{\theta a} q_{T}(a ; \tilde{c}) d a n_{T}(z) d z\right)\right],
\end{aligned}
$$

where the densities $p(u ; \tilde{c})$ and $q_{T}(a ; \tilde{c})$ are given by (25) and (30) respectively, the functions $\Psi_{T^{ \pm}}^{ \pm}$are given by $(29,31,32,33), g(a, b, \gamma, T-u)$ represents the density 
of the triplet involved in each case, and the domain $D^{ \pm}$is $(-\infty, \tilde{c})(\operatorname{resp} .(\tilde{c}, \infty))$ if $\tilde{c}>0$ (resp. $\tilde{c}<0)$.

Using Fubini between $u$ and $\gamma$, the fact that $\Psi_{T^{-}}^{ \pm}$is independent of $u$, and the convolution relation

$$
\begin{aligned}
& \int_{0}^{T-\gamma} g(a, b, \gamma ; T-u) p(u ; \tilde{c}) d u \\
& =\left\{\begin{array}{lll}
2 p(\gamma ; a+b) p(T-\gamma ; b+|\tilde{c}|) & \text { if } & a>0, \\
2 p(\gamma ; b) p(T-\gamma ;-a+b+|\tilde{c}|) & \text { if } & a<0,
\end{array}\right. \\
& =: G(a, b, \gamma ; T),
\end{aligned}
$$

we arrive at the pricing formula:

$$
\begin{aligned}
P_{0}= & e^{-\left(r+\frac{1}{2} \theta^{2}\right) T}\left[e^{\theta \tilde{c}} \int_{-\infty}^{\infty} \int_{0}^{T} \int_{0}^{\infty} \int_{-\infty}^{\infty} h\left(e^{\Psi_{T^{-}}^{ \pm}(a, b, \gamma, z)}\right) e^{\theta a}\right. \\
& \times G(a, b, \gamma ; T) d a d b d \gamma n_{T}(z) d z \\
& \left.+\left(\int_{-\infty}^{\infty} \int_{D^{ \pm}} h\left(e^{\Psi_{T^{+}}^{ \pm}(a, z)}\right) e^{\theta a} q_{T}(a ; \tilde{c}) d a n_{T}(z) d z\right)\right]
\end{aligned}
$$

Numerically, one has to consider separately the two cases $\tilde{c}>0$ and $\tilde{c}<0$ which determine the choice of functions $\Psi^{ \pm}$given by $(29,31,32,33)$. One also must be careful in each case to decompose the integral with respect to $a$ over $a>0$ and $a<0$ since the function $G$ given by (34) depends on it. Recall that the densities $p$ and $q_{T}$ are given by (25) and (30) respectively, and

$$
D^{ \pm}=\left\{\begin{array}{lll}
(-\infty, \tilde{c}) & \text { if } & \tilde{c}>0 \\
(\tilde{c}, \infty) & \text { if } & \tilde{c}<0
\end{array}\right.
$$

\section{Remarks}

1. If $\tilde{c}=0$ (equivalently $\xi=\log c$ or $M_{0}=c$ ), then $\tau=0$ and the pricing formula (35) reduces to:

$$
\begin{aligned}
P_{0}= & e^{-\left(r+\frac{1}{2} \theta^{2}\right) T} \\
& \times \int_{-\infty}^{\infty} \int_{0}^{T} \int_{0}^{\infty} \int_{-\infty}^{\infty} h\left(e^{\Psi_{0}(a, b, \gamma, z)}\right) e^{\theta a} G(a, b, \gamma ; T) d a d b d \gamma n_{T}(z) d z
\end{aligned}
$$

where the function $\Psi_{0}(a, b, \gamma, z)$ was introduced in (27). 
2. If $\delta=0$ (for any value of $\tilde{c}$ ), the pricing formula (35) reduces to the Black-Scholes pricing formula with square-volatility $\sigma_{m}^{2} \beta^{2}+\sigma^{2}$ as it should be since that case corresponds to the linear CAPM model described in Sect. 2.1. This reduction is actually not straightforward. One needs to remark that $A_{2}=0$, and $a+\tilde{c}$ recombines, so that $\Psi_{ \pm}$becomes $x+A_{1} T+\sigma_{m} w+\sigma z$.

3. For hedging purposes, one needs to compute the Deltas given by (12). They are obtained by taking derivatives of the option price (35) with respect to $\xi$ and $x$. Note that $x$ appears only in the payoff function $h$ (specifically in the functions $\Psi^{ \pm}$), while $\xi$ appears also in the density functions $G$ and $q_{T}$, and in the domain of integration, making the corresponding formula more complicated and numerically involved.

\section{Market volatility}

In the model described by the dynamics (1)-(2) under the physical measure, the market volatility $\sigma_{m}$ is assumed to be constant. This is indeed not realistic both from the point of view of the market returns distribution and the market skews of implied volatilities. In this section, we propose to introduce stochastic volatility in the market model. We will follow the approach taken in Fouque et al. (2000) and also used recently in Fouque and Kollman (2009) in the CAPM context. In this generalized CAPM model, the market volatility is driven by a fast mean-reverting factor according to

$$
\begin{aligned}
\frac{d M_{t}}{M_{t}} & =\mu d t+f\left(Y_{t}\right) d W_{t}, \\
d Y_{t} & =\frac{1}{\epsilon}\left(m-Y_{t}\right) d t+\frac{v \sqrt{2}}{\sqrt{\epsilon}} d W_{t}^{y}, \\
\frac{d S_{t}}{S_{t}} & =\beta\left(M_{t}\right) \frac{d M_{t}}{M_{t}}+\sigma d Z_{t},
\end{aligned}
$$

where $Y_{t}$ is an Ornstein-Uhlenbeck (OU) process with large rate of mean-reversion $1 / \varepsilon$, that is $\varepsilon$ is a small positive parameter, and which admits the Gaussian invariant distribution $\mathcal{N}\left(m, v^{2}\right)$. The function $f$ is positive increasing, which can be assumed smooth bounded and bounded away from zero for technical simplicity. The Brownian motions $W_{t}$ and $W_{t}^{y}$ are correlated according to $d\left\langle W, W^{y}\right\rangle_{t}=\rho d t$ where $\rho$ is constant with $|\rho|<1$. The equation for the asset price $S_{t}$ is unchanged, and the Brownian motion $Z_{t}$ is independent of $W_{t}$ and $W_{t}^{y}$. As before, the function $\beta(M)$ is given by (5).

\subsection{Risk-neutral pricing measure}

As before, the market and the asset being both tradable, their discounted prices need to be martingales under a risk-neutral pricing measure. In order to achieve that, we first write 


$$
W_{t}^{y}=\rho d W_{t}+\sqrt{1-\rho^{2}} d W_{t}^{\perp},
$$

with now $\left(W_{t}, W_{t}^{\perp}, Z_{t}\right)$ being three independent standard Brownian motions, and then we rewrite the system $(37,38,39)$ as:

$$
\begin{aligned}
\frac{d M_{t}}{M_{t}}= & r d t+f\left(Y_{t}\right)\left(d W_{t}+\frac{\mu-r}{f\left(Y_{t}\right)} d t\right) \\
d Y_{t}= & \frac{1}{\epsilon}\left(m-Y_{t}\right) d t-\frac{\nu \sqrt{2}}{\sqrt{\varepsilon}} \Lambda\left(Y_{t}\right) d t \\
& +\frac{\nu \sqrt{2}}{\sqrt{\epsilon}}\left[\rho\left(d W_{t}+\frac{\mu-r}{f\left(Y_{t}\right)} d t\right)+\sqrt{1-\rho^{2}}\left(d W_{t}^{\perp}+\gamma\left(Y_{t}\right) d t\right)\right], \\
\frac{d S_{t}}{S_{t}}= & r d t+\beta\left(M_{t}\right) f\left(Y_{t}\right)\left(d W_{t}+\frac{\mu-r}{f\left(Y_{t}\right)} d t\right)+\sigma\left(d Z_{t}+\frac{(\beta-1) r}{\sigma} d t\right),
\end{aligned}
$$

where $\gamma\left(Y_{t}\right)$ is a market price of volatility risk, which we suppose to depend on $Y_{t}$ only, and we define

$$
\Lambda\left(Y_{t}\right)=\rho \frac{\mu-r}{f\left(Y_{t}\right)}+\sqrt{1-\rho^{2}} \gamma\left(Y_{t}\right)
$$

Setting

$$
\begin{aligned}
d W_{t}^{*} & =d W_{t}+\frac{\mu-r}{f\left(Y_{t}\right)} d t, \\
d W_{t}^{\perp *} & =d W_{t}^{\perp}+\gamma\left(Y_{t}\right) d t, \\
d Z_{t}^{*} & =d Z_{t}+\frac{(\beta-1) r}{\sigma} d t,
\end{aligned}
$$

by Girsanov theorem, there is an equivalent probability $\mathbb{P}^{\star}(\gamma)$ such that $\left(W_{t}^{*}, W_{t}^{\perp *}\right.$, $\left.Z_{t}^{*}\right)$ are independent standard Brownian motions under $\mathbb{P}^{\star}(\gamma)$, called the pricing equivalent martingale measure and determined by the market price of volatility risk $\gamma$. We assume here that both the Sharpe ratio $\frac{\mu-r}{f\left(Y_{t}\right)}$ and $\gamma\left(Y_{t}\right)$ are bounded, which, depending on the choice of function $f$, may require that $\mu$ depends on $Y_{t}$. Finally, under $\mathbb{P}^{\star}(\gamma)$, the dynamics $(37,38,39)$ becomes:

$$
\begin{aligned}
\frac{d M_{t}}{M_{t}} & =r d t+f\left(Y_{t}\right) d W_{t}^{*}, \\
d Y_{t} & =\frac{1}{\epsilon}\left(m-Y_{t}\right) d t-\frac{\nu \sqrt{2}}{\sqrt{\varepsilon}} \Lambda\left(Y_{t}\right) d t+\frac{\nu \sqrt{2}}{\sqrt{\epsilon}} d W_{t}^{y *}, \\
W_{t}^{y *} & =\rho W_{t}^{*}+\sqrt{1-\rho^{2}} W_{t}^{\perp *}, \\
\frac{d S_{t}}{S_{t}} & =r d t+\beta\left(M_{t}\right) f\left(Y_{t}\right) d W_{t}^{*}+\sigma d Z_{t}^{*} .
\end{aligned}
$$


In what follows, we take the point of view that by pricing options on the index $M$ and on the particular asset $S$, the market is "completing" itself and indirectly choosing the market price of volatility risk $\gamma$.

\subsection{Market option prices}

In looking at option prices on the market index we only focus on the autonomous evolution of $\left(M_{t}, Y_{t}\right)$ described by equations $(40,41)$ under the risk-neutral pricing measure. A singular perturbation approach to option pricing on the model described in $(40,41)$ was developed in Fouque et al. (2000). Here we use this approximation technique but with an additional parameter reduction to allow for parameter estimation using option data only. The details of this derivation can be found in the appendix A of Fouque and Kollman (2009), and lead to the following price approximation for call options on the market. Let $P^{\epsilon}=P(t, M, y ; T, K)$ denote the price of a European call option written on the market index $M$, with maturity $T$ and strike $K$, evaluated at time $t<T$ with current values $M_{t}=M$ and $Y_{t}=y$, where we explicitly show the dependence on the small volatility mean-reversion time $\varepsilon$. Then, we have the following approximation

$$
P^{\epsilon} \sim P^{*}+(T-t) V_{3}^{\epsilon} M \frac{\partial}{\partial M}\left(M^{2} \frac{\partial^{2} P^{*}}{\partial M^{2}}\right)
$$

where $P^{*}=P_{B S}\left(\sigma^{*}\right)$ is the corresponding Black Scholes call price with constant volatility equal to the adjusted effective volatility $\sigma^{*}$. Here

$$
\sigma^{*}=\sqrt{\bar{\sigma}^{2}+2 V_{2}^{\varepsilon}}
$$

where $\bar{\sigma}$ is the effective volatility defined by

$$
\bar{\sigma}^{2}=\left\langle f^{2}\right\rangle \equiv \int f(y)^{2} \frac{1}{\sqrt{2 \pi} v} e^{-\frac{(y-m)^{2}}{2 v^{2}}} d y,
$$

with the average being taken with respect to the invariant distribution of the OU process $Y$. The small parameter $V_{2}^{\varepsilon}$-which is proportional to $\sqrt{\epsilon}$ and arises in the asymptotic analysis - accounts for a volatility adjustment due to the market price of volatility risk. The small parameter $V_{3}^{\varepsilon}$ appearing in (43) is proportional to $\sqrt{\epsilon}$ and to the correlation coefficient $\rho$, and it accounts for the skew of implied volatility. It is shown in Fouque et al. (2003) that the accuracy of the approximation (43) is $\mathcal{O}(\varepsilon \log |\varepsilon|)$. 
5.3 Market implied volatilities

Following Fouque et al. (2000) and Fouque and Kollman (2009), we introduce the Log-Moneyness to Maturity Ratio (LMMR)

$$
L M M R=\frac{\log (K / x)}{T},
$$

and for calibration purposes, we use the affine LMMR formula

$$
I \sim b^{*}+a^{\epsilon} L M M R
$$

with the intercept $b^{*}$ and the slope $a^{\varepsilon}$ to be fitted to the skew of option data. We then use the estimators derived in Fouque and Kollman (2009):

$$
\begin{gathered}
\sigma^{*} \sim b^{*}+a^{\epsilon}\left(r-\frac{b^{* 2}}{2}\right) \equiv \widehat{\sigma^{*}}, \\
V_{3}^{\epsilon}=a^{\epsilon} \sigma^{* 3} \sim a^{\epsilon} b^{* 3} \equiv \widehat{V_{3}^{\varepsilon}} .
\end{gathered}
$$

\subsection{Effect on asset options}

Indeed, the introduction of market stochastic volatility in the model has also an effect on the dynamics of the asset price (42) where the constant volatility $\sigma_{m}$ in (10) has been replaced by $f\left(Y_{t}\right)$. However, the asymptotic analysis performed on asset option prices as in Fouque and Kollman (2009) reveals that, to the leading order, these prices are given by $(9,10,11)$ with $\sigma_{m}$ replaced by $\sigma^{*}$ given by (44) and calibrated on market skews using (48). Therefore, in what follows, we simply use our pricing formula (35) with $\sigma_{m}$ replaced by $\sigma^{*}$.

Note that one could derive a formula for the first order correction due to market stochastic volatility which would involve the parameter $V_{3}^{\varepsilon}$ appearing in (43) and calibrated using (49). However, this formula is quite complicated and numerically involved, and here, for the purpose of calibration of $\beta$ and $\delta$ (the main goal of this paper), we restrict ourselves to the leading order.

\section{Numerical results and calibration}

\subsection{Asset skews of implied volatilities}

This section presents implied volatility skews produced from the Stressed-Beta model. Additionally, a sensitivity analysis examines how the implied volatility skew responds to a change in the model parameter $\delta$ or the initial value $M_{0}$. For each of these studies, a European call option is priced, and the following parameter settings are used: $c=1,000, S_{0}=100, r=0.01, \beta=1, \sigma_{m}=0.30, \sigma=0.01$, and $T=1$. Strike prices of $70,71, \ldots, 150$ are used to build the implied volatility curves. 
For the sensitivity analysis with respect to $\delta$, we consider the case when $M_{0}=c$, that is when the first passage time occurs at the start. In this case, the log-stock price $X_{T}$ at terminal time is given by (27) and call options are priced according to the simplified formula (36).

For the sensitivity with respect to $M_{0}$, we examine the implied volatility skew produced from a starting market price that is (1) far below the boundary $c$, (2) below the boundary, (3) at the boundary, (4) above the boundary, and (5) far above the boundary. The value of $\delta$ is set to 0.5 for this analysis.

Figure 1 illustrates that for each setting of $\delta$, the implied volatility curve exhibits a skew. Implied volatility is an increasing function of $\delta$ for each moneyness, and the slope of the volatility skew is an increasing function of $\delta$ as well.

Next, consider the results from the $M_{0}$ study, shown in Fig. 2. When the market price starts far below the boundary $\left(M_{0}=500\right)$, it is very unlikely that it will cross above $c$, and thus asset volatility will most likely remain at the high setting through expiry. This is the Black-Scholes model with volatility $\sqrt{(\beta+\delta)^{2} \sigma_{m}^{2}+\sigma^{2}}=0.4501$, which is an upper bound. Note that the implied volatility curve for $M_{0}=500$ is approximately equal to this value. As $M_{0}$ decreases further, the Stressed-Beta price of the option will converge to the Black-Scholes price with high volatility. Next, consider the case when the market price starts far above the boundary $\left(M_{0}=2000\right)$. Now, it is very unlikely that the market price will cross below $c$, and so the asset volatility will most likely remain at the low setting through expiry. This is the Black-Scholes model with volatility $\sqrt{\beta^{2} \sigma_{m}^{2}+\sigma^{2}}=0.3002$. The implied volatility curve for $M_{0}=2000$ is equal to this value, which forms the lower bound on implied volatility. For a value of $M_{0}$ closer to $c$, the implied volatility curve exhibits a skew, and it falls within the interval [0.3002, 0.4501]. This skew, in fact, will be greatest at $M_{0}=c$, and will flatten as $M_{0}$ is moved away from the boundary.

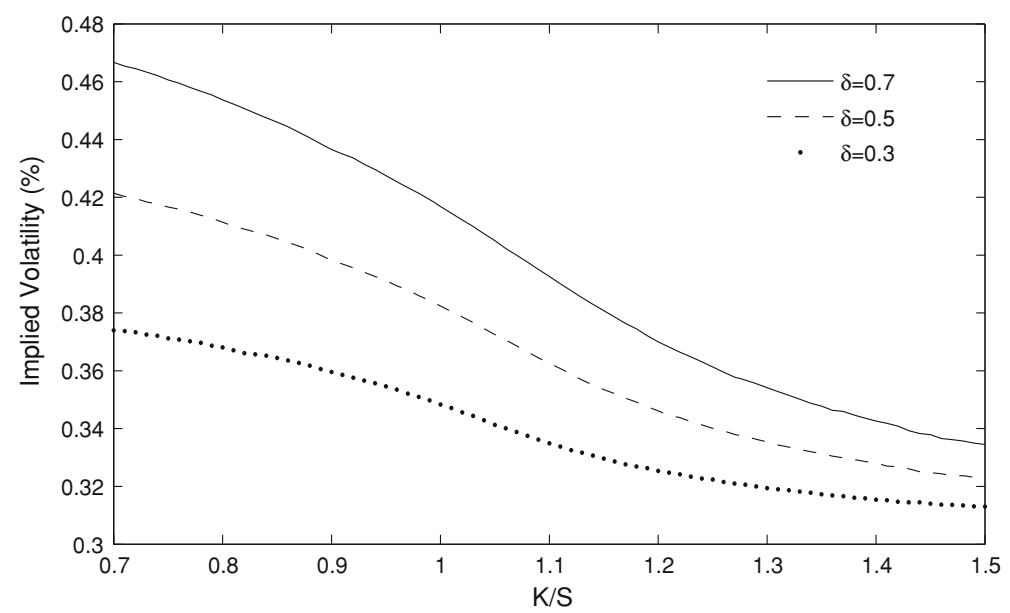

Fig. 1 Implied volatility versus moneyness of a European call option for different values of $\delta$. The level $c$ is fixed at $1,000, S_{0}=100, T=1, r=0.01, \beta=1, \sigma_{m}=0.30$, and $\sigma=0.01$. The starting market price $M_{0}$ is set equal to $c=1,000$ 


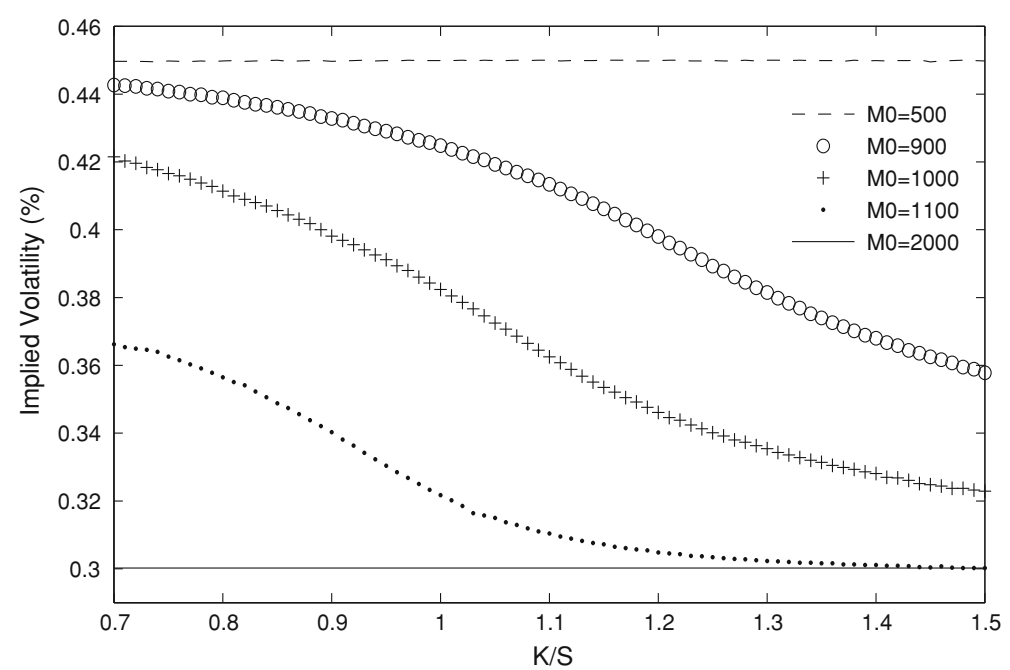

Fig. 2 Implied volatility versus moneyness of a European call option for different values of the starting market price, $M_{0}$. The level $c$ is fixed at $1,000, S_{0}=100, T=1, r=0.01, \beta=1, \delta=0.5, \sigma_{m}=0.30$, and $\sigma=0.01$

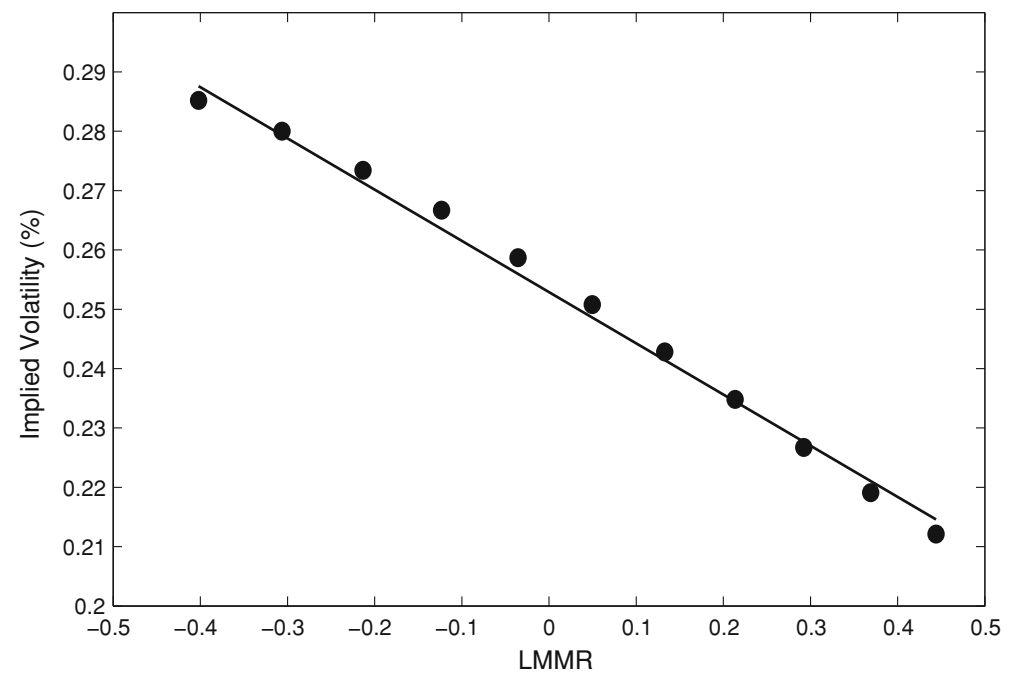

Fig. 3 Affine LMMR fit to S\&P 500 Index options expiring September 18, 2009 based on May 26, 2009 market prices. The estimated slope is $a^{\varepsilon}=-0.086$, the estimated intercept is $b^{*}=0.25$, and the $R$-squared is 0.9741

\subsection{Calibration to data}

Next, we calibrate the Stressed-Beta model to Amgen options with October 2009 expiry. We consider options with $\operatorname{LMMR}$ (46) between -1 and 1 , using closing mid-prices as of May 26, 2009. For simplicity, here we set to zero the asset-specific 


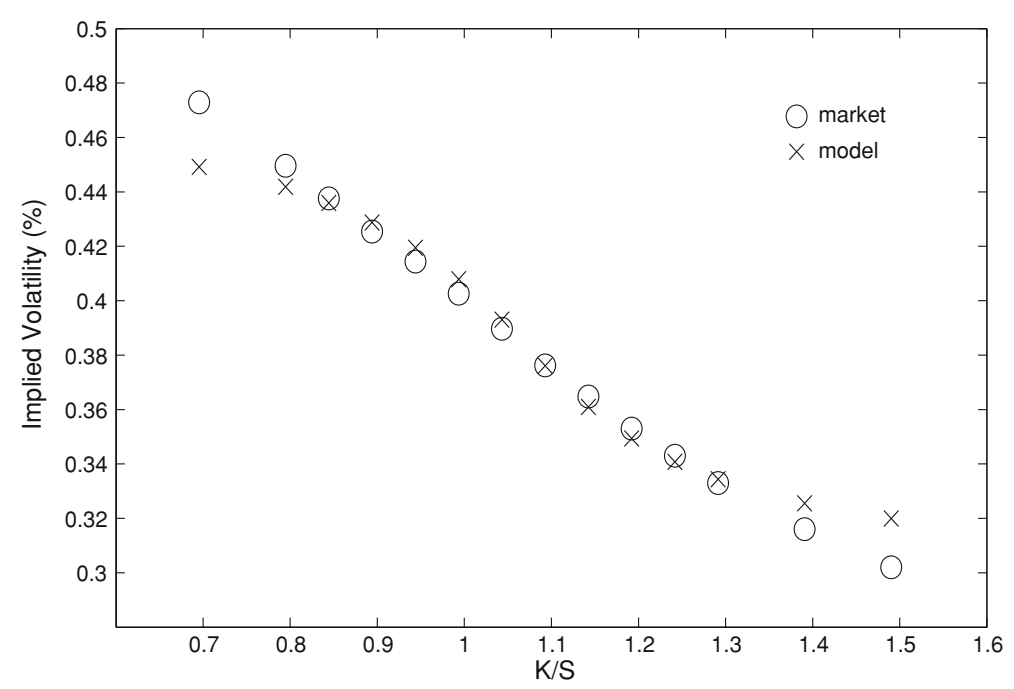

Fig. 4 Volatility skews for Amgen call options expiring October 2009 based on (1) market prices as of May 26, 2009 and (2) the Stressed-Beta model. The model parameters are the following: $c=925, \beta=1.17$, $\delta=0.65, \sigma^{*}=0.2549$, and $\sigma=0$

volatility $\sigma$. The market volatility $\sigma^{*}$ is estimated from option data on the S\&P 500 Index. We use options with strike prices between 800 and 1,200, and expiry closest to October 2009 (these are the September-expiry options). Next, the affine LMMR formula (47) is fit to the skew of the option data as shown in Fig. 3. Given the slope, intercept, and 4-month-interpolated LIBOR rate, $\sigma^{*}=0.2549$ is obtained from (48). There are now three free parameters: $c, \beta$, and $\delta$. These parameters are set to the values which minimize the sum of squared errors between the option model prices and market prices. As this procedure is computationally intensive, we limit the search across $c$ to the following set of values: $900,925,950,975,1,000$. The closing level of the S\&P 500 Index as of May 26, 2009 was 910.33.

Figure 4 shows the fit of our model to this set of option data. The estimated parameters are: $c=925, \beta=1.17$, and $\delta=0.65$.

Open Access This article is distributed under the terms of the Creative Commons Attribution Noncommercial License which permits any noncommercial use, distribution, and reproduction in any medium, provided the original author(s) and source are credited.

\section{References}

Akdeniz, L., Salih, A.A., Caner, M.: Time-varying betas help in asset pricing: the threshold CAPM. Stud Nonlinear Dyn Econ 6, 4 (2003)

Ferson, W.E.: Changes in expected security returns, risk, and the level of interest rates. J Financ 44(5), 11911214 (1989)

Ferson, W.E., Harvey, C.R.: The variation of economic risk premiums. J Polit Econ 99(2), 385-415 (1991)

Ferson, W.E., Harvey, C.R.: The risk and predictability of international equity returns. Rev Financial Stud 6(3), 527-566 (1993) 
Ferson, W.E., Korajczyk, R.A.: Do arbitrage pricing models explain the predictability of stock returns? J Bus 68(3), 309-349 (1995)

Fouque, J.-P., and Kollman, E.: Calibration of stock betas from skews of implied volatilities. Submitted (2009)

Fouque, J.-P., Papanicolaou, G., Sircar, R.: Derivatives in Financial Markets with Stochastic Volatility. Cambridge University Press, Cambridge (2000)

Fouque, J.-P., Papanicolaou, G., Sircar, R.K. Sølna: Singular perturbations in option pricing. SIAM J Appl Math 63(5), 1648-1665 (2003)

Fridman, M.: A Two state capital asset pricing model. IMA Preprint series \#1221 (1994)

Ghysels, E.: On stable factor structures in the pricing of risk: do time varying betas help or hurt? J Financ 53(2), 549-573 (1998)

Heston, S.: A closed-form solution for options with stochastic volatility with applications to bonds and currency options. Rev Financial Stud 6(2), 327-343 (1993)

Jagannathan, R., Wang, Z.: The conditional CAPM and the cross-section of expected returns. J Financ 51(1), 3-53 (1996)

Karatzas, I., Shreve, S.E.: Trivariate density of brownian motion, its local and occupation times, with application to stochastic control. Ann Probab 12(3), 819-828 (1984)

Karatzas, I., Shreve, S.E.: Brownian Motion and Stochastic Calculus, 2nd edn. Springer, Berlin (1991)

Lintner, J.: The valuation of risky assets and the selection of risky investments in stock portfolio and capital budgets. Rev Econ Stat 47(1), 13-37 (1965)

Markowitz, H.: Portfolio selection. J Financ 7(1), 77-99 (1952)

Mossin, J.: Equilibrium in a capital asset market. Econometrica 34(4), 768-783 (1966)

Sharpe, W.F.: Capital asset prices: a theory of market equilibrium under conditions of risk. J Financ 19(3), 425-442 (1964)

Treynor, J.L.: Toward a theory of market value of risky assets. Unpublished manuscript. (1962).

Tashman, A.P., Frey, R.J.: Modeling risk in arbitrage strategies using finite mixtures. Quant Financ 9(5), 495503 (2009)

Tashman, A.P.: Dynamic risk management of a Hedge fund in a regime-switching environment. Submitted (2009) 